\title{
Approximate solution of boundary integral equations for biharmonic problems in non-smooth domains
}

\author{
Victor Didenko ${ }^{1, *}$ and Johan Helsing ${ }^{2, * *}$ \\ ${ }^{1}$ Faculty of Science, University of Brunei Darussalam, Bandar Seri Begawan, BE1410 Brunei \\ ${ }^{2}$ Centre for Mathematical Sciences, Lund University, Box 118, SE-221 00 Lund, Sweden
}

\begin{abstract}
This paper deals with approximate solutions to integral equations arising in boundary value problems for the biharmonic equation in simply connected piecewise smooth domains. The approximation method considered demonstrates excellent convergence even in the case of boundary conditions discontinuous at corner points. In an application we obtain very accurate approximations for some characteristics of two-dimensional Stokes flow in non-smooth domains.
\end{abstract}

Copyright line will be provided by the publisher

\section{Introduction}

Let $D$ be a domain in the complex plane $\mathbb{C}$ bounded by a simple counterclockwise oriented contour $\Gamma$. It is well-known that various problems of elasticity and fluid dynamics can be formulated as the biharmonic equation

$$
\Delta^{2} \mathbf{U}(x, y)=0, \quad(x, y) \in D
$$

where $\Delta:=\partial^{2} / \partial x^{2}+\partial^{2} / \partial y^{2}$ is the Laplace operator, and $\mathbf{U}$ satisfies certain conditions on the boundary $\Gamma$. On the other hand, this equation can be reduced to one of two famous integral equations, viz. to the Sherman-Lauricella

$$
A_{S L} \omega(t):=\omega(t)+\frac{1}{2 \pi i} \int_{\Gamma} \omega(\tau) d \ln \left(\frac{\tau-t}{\bar{\tau}-\bar{t}}\right)-\frac{1}{2 \pi i} \int_{\Gamma} \overline{\omega(\tau)} d\left(\frac{\tau-t}{\bar{\tau}-\bar{t}}\right)=f(t), \quad t \in \Gamma,
$$

or to the Muskhelishvili equation

$$
A_{M} \varphi(t):=-\overline{\varphi(t)}-\frac{1}{2 \pi i} \int_{\Gamma} \overline{\varphi(\tau)} d \ln \left(\frac{\bar{\tau}-\bar{t}}{\tau-t}\right)-\frac{1}{2 \pi i} \int_{\Gamma} \varphi(\tau) d\left(\frac{\bar{\tau}-\bar{t}}{\tau-t}\right)=f_{0}(t), \quad t \in \Gamma,
$$

where the right-hand sides $f$ and $f_{0}$ are functions generated by the corresponding boundary conditions. Each of these equations has its own merits and is used depending on the problem considered [1-3]. However, numerical approaches may not directly apply to either of the equations (1) or (2) because the operators $A_{S L}$ and $A_{M}$ of (2)-(3) are not invertible in the main functional spaces. Thus any direct projection method for (2) or (3) is unstable. Therefore, these equations have to be corrected in a suitable way. For example, one can add an operator $T_{S L}$ or $T_{M}$ to obtain invertible operators $A_{S L}+T_{S L}$ or $A_{M}+T_{M}$ such that the solution of the corresponding corrected equation will be a solution of the original equation, cf. [4-6]. Further, if the boundary $\Gamma$ has corner points, the integral operators in (2)-(3) are not compact. As a result, the approximation method used is not always stable and certain stability conditions may be needed.

In the present paper we consider quadrature methods for the Sherman-Lauricella equation (2). In particular, necessary and sufficient conditions of the stability of the original Nyström method are presented. It turns out that the method is stable if and only if certain operators associated with the corner points of $\Gamma$ and with the corresponding approximation method are invertible. Moreover, we derive an approximate solution of the associated equation (2) for boundary value problems for the biharmonic equation in the unit square and use it to describe the related Stokes flow.

\section{Quadrature methods for the Sherman-Lauricella Equation}

Let $\gamma=\gamma(s), s \in \mathbb{R}$ be a 1-periodic parametrization of $\Gamma$, and let $c_{0}, c_{1}, \ldots, c_{q-1}$ be the corner points of $\Gamma$. Assuming that $c_{j}=\gamma(j / q), j=0,1, \ldots, q-1$, one can take two sets of real numbers $0<\varepsilon_{0}<\varepsilon_{1}<\ldots<\varepsilon_{d-1}<1$ and $0<\delta_{0}<\delta_{1}<\ldots<\delta_{d-1}<1$, and consider the following points on $\Gamma$,

$$
\tau_{k r}=\gamma\left(\frac{k+\varepsilon_{r}}{n}\right), \quad t_{k r}=\gamma\left(\frac{k+\delta_{r}}{n}\right), \quad k=0,1, \ldots, n-1 ; r=0,1, \ldots, d-1,
$$

* Corresponding author: e-mail diviol@ gmail.com, phone +6732229995

** Corresponding author: e-mail helsing@ maths.lth.se, phone +46462223372 
Approximate values of an exact solution $\omega\left(\tau_{k r}\right)$ of (2) at the points $\tau_{k r}$ can be obtained from the system of algebraic equations

$$
\begin{aligned}
\omega\left(\tau_{k r}\right) & +\frac{1}{2 \pi i} \sum_{l=0}^{n-1} \sum_{p=0}^{d-1} w_{p} \omega\left(\tau_{l p}\right)\left(\frac{\tau_{k r}^{\prime}}{\tau_{l p}-t_{k r}}-\frac{{\overline{\tau^{\prime}}}_{l p}}{\bar{\tau}_{l p}-\bar{t}_{k r}}\right) \frac{1}{n} \\
& -\frac{1}{2 \pi i} \sum_{l=0}^{n-1} \sum_{p=0}^{d-1} w_{p} \overline{\omega\left(\tau_{l p}\right)}\left(\frac{1}{\bar{\tau}_{l p}-\bar{t}_{k r}} \frac{\tau_{l p}^{\prime}}{n}-\frac{\tau_{l p}-t_{k r}}{\left(\bar{\tau}_{l p}-\bar{t}_{k r}\right)^{2}} \frac{\overline{\tau^{\prime}}}{n}\right) \\
& +\frac{1}{\left(\bar{t}_{k r}-\bar{a}\right)} \frac{1}{2 \pi i} \sum_{l=0}^{n-1} \sum_{p=0}^{d-1} w_{p}\left(\frac{\omega_{l p}}{\left(\tau_{l p}-a\right)^{2}} \frac{\tau_{l p}^{\prime}}{n}+\frac{\overline{\omega\left(\tau_{l p}\right)}}{\left(\bar{\tau}_{l p}-\bar{a}\right)^{2}} \frac{\overline{\tau^{\prime}} l p}{n}\right)=f\left(t_{k r}\right) \\
k & =0,1, \ldots, n-1 ; r=0,1, \ldots, d-1,
\end{aligned}
$$

where $\tau_{l p}^{\prime}=\gamma^{\prime}\left(\left(l+\varepsilon_{p}\right) / n\right)$ and $a$ is an arbitrary fixed point in $D$. Having obtained the values $\omega\left(\tau_{k r}\right)$ and using splines of order $d$, one can easily construct an approximate solution of equation (2) coinciding with the approximate values of the solution $\omega$ at the points $\omega\left(\tau_{k r}\right), k=0,1, \ldots, n-1 ; r=0,1, \ldots, d-1$. Let us denote this solution by $\omega_{n}=\omega_{n}(t), t \in \Gamma$.

For a corner point $c_{j} \in \Gamma$, let $\theta_{j}, 0<\theta_{j}<2 \pi$ be the left angle between the right and left semi-tangents to $\Gamma$ at $c_{j}$. Consider the operators $B_{\theta_{j}, \delta, \varepsilon}$ defined on the space $l^{2}$ by [6, Formula (3.9)]. It was noted in [7] that there is at most countable set $\mathfrak{S}_{c}$ of opening angles $\theta_{j} \in(0,2 \pi)$, called critical angles, such that the operator $B_{\theta_{j}, \delta, \varepsilon}$ is not invertible.

Proposition 2.1 Let $f$ belong to the Sobolev space $W_{2}^{1}(\Gamma)$ and let

$$
\operatorname{Re} \int_{\Gamma} \overline{f(t)} d t=0
$$

The systems (4) are solvable for all sufficiently large $n=m q, m \in \mathbb{N}$ and the approximate solutions $\omega_{n}$ converge to a solution of equation (2) in the norm $L^{2}(\Gamma)$ if and only if for any $j=0,1, \ldots, q-1$ the value $\theta_{j}$ does not belong to the set $\mathfrak{S}_{c}$.

The proof of this proposition follows from [6], [7].

Remark 2.2 The right-hand sides $f$ arising in applications are not always continuous. However, the Sobolev space $W_{2}^{1}(\Gamma)$ in Proposition 2.1 can be replaced by the space $W_{2}^{1}\left(\Gamma, c_{0}, c_{1}, \ldots, c_{q-1}\right)$ of functions which belong to $W_{2}^{1}$ on each arc $\left(c_{j}, c_{j+1}\right)$ but may be discontinuous at corner points $c_{j}$.

Example 2.3 Consider the Sherman-Lauricella equation (2) where $\Gamma$ is the boundary of the unit square centered at the origin and the right-hand side $f$ is defined by

$$
f(t)=f(x, y)=\left\{\begin{aligned}
-y & \text { if } \quad x=0.5,-0.5<y<0.5 \\
i & \text { if } y=0.5,-0.5<x<0.5 \\
-y & \text { if } x=-0.5,-0.5<y<0.5 \\
0 & \text { if } y=-0.5,-0.5<x<0.5
\end{aligned}\right.
$$

Note that $f$ has four discontinuity points, all located at the corner points of $\Gamma$. Nevertheless, the approximation method (4) has a very high convergence rate. Moreover, since $f \in W_{2}^{1}\left(\Gamma, c_{0}, c_{1}, c_{2}, c_{3}\right)$ and condition (5) is satisfied, the approximate solutions $\omega_{n}$ converge to an exact solution of the corresponding equation (2). Besides, all opening angles $\theta_{j}$ of the corner points are equal to $\pi / 2$, and by [7] the angle $\pi / 2 \notin \mathfrak{S}_{c}$. Therefore, the above method is stable and converges for any right hand side from the above-mentioned space. The corresponding approximate solution is shown in Fig. 1.

\section{Numerical Examples: Stokes flow in planar domains with corner points}

In the two-dimensional case, the stream function $\mathbf{U}=\mathbf{U}(x, y),(x, y) \in \mathbb{R}^{2}$ representing an incompressible Stokes flow at some instant satisfies the biharmonic equation (1). Thus if $\mathbf{U}$ is known, the velocity field $(u, v)$ and vorticity $\zeta$ can be established - viz. $(u, v)=(\partial \mathbf{U} / \partial y,-\partial \mathbf{U} / \partial x), \zeta:=u_{y}-v_{x}$. On the other hand, various boundary value problems for the biharmonic equation (1) can be reduced to to the boundary value problem

$$
\varphi(t)+\overline{\varphi^{\prime}(t)}+\overline{\psi(t)}=f(t), \quad t \in \Gamma
$$

for two functions $\varphi$ and $\psi$ holomorphic in the domain $D$. By [1], the solution of the last problem is representable in the form

$$
\varphi(z)=\frac{1}{2 \pi i} \int_{\Gamma} \frac{\omega(\tau)}{\tau-z} d \tau, \quad \psi(z)=\frac{1}{2 \pi i} \int_{\Gamma} \frac{\overline{\omega(\tau)} d \tau+\omega(\tau) d \bar{\tau}}{\tau-z}-\frac{1}{2 \pi i} \int_{\Gamma} \frac{\bar{\tau} \omega(\tau)}{(\tau-z)^{2}} d \tau, \quad z \in D,
$$

where $\omega$ is the solution of the Sherman-Lauricella equation (2), and the biharmonic function in question is given by

$$
\mathbf{U}(x, y):=\operatorname{Re}(\bar{z} \varphi(z)+\chi(z)), \quad \chi^{\prime}(z)=\psi(z), \quad z=x+i y \in D .
$$



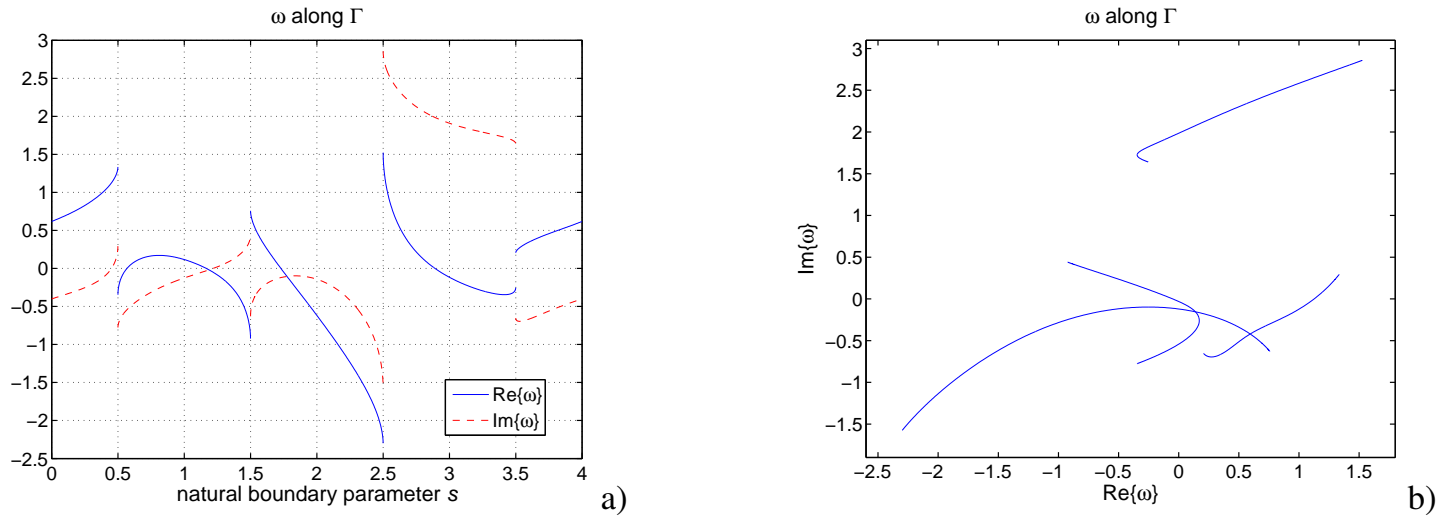

Fig. 1: Solution $\omega$ to (2) with a discontinuous $f$ given by (6). a shows $\omega$ as a function of arc length $s$. b shows $\omega$ in the complex plane.

Thus the main characteristics of two-dimensional Stokes flow can be obtained from the solution of the Sherman-Lauricella equation (2) via formulas (8)-(9) with an appropriately constructed right-hand side $f$. Moreover, in order to approximate the components $u$ and $v$ of the velocity vector and the vorticity $\zeta$ one can use more convenient representations

$$
u(x, y)+i v(x, y)=\varphi(z)+z \overline{\varphi^{\prime}(z)}+\overline{\psi(z)}, \quad \zeta=4 \operatorname{Re} \varphi^{\prime}(z), \quad z=x+i y \in D
$$

instead of direct use of the expression (9). The first formula (10) can be found e.g. in [5, Page 236], and the second can be derived analogously.

From now on, let $D$ refer to the unit square $D:=\left\{(x, y) \in \mathbb{R}^{2}:-0.5<x<0.5,-0.5<y<0.5\right\}$, whose boundary $\Gamma$ is given the natural parametrization $\gamma=\gamma(s)$ with starting point $\gamma(0)=(-0.5,0)$. Choose $d=16$ in (4) and let $\left\{\epsilon_{r}\right\}$ and $\left\{\delta_{r}\right\}$ both coincide with the zeros of the Legendre polynomial $P_{16}(x)$, scaled and shifted to the interval $x \in[0,1]$. This corresponds to the original Nyström method with composite 16-point Gauss-Legendre quadrature. Further, let the quadrature mesh on $\Gamma$ be refined in a direction towards the corner points as deemed appropriate. On the subintervals neighbouring the corner points, the Gauss-Legendre quadrature is replaced by interpolatory quadrature (product integration) using techniques from [8, Section 4$]$ and [9, Section 2]. The same mix of Gauss-Legendre quadrature and an interpolatory quadrature is used in the discretization of the integrals for velocity and vorticity. The linear system (4) is solved using techniques from [10]. For error estimates, we compare the analytic and approximate solutions if the former is available. Otherwise, we compare successive numerical solutions produced via uniformly overresolved meshes.

Example 3.1 In the domain $D$ consider the biharmonic problem (1) with the following boundary conditions

$$
\frac{\partial \mathbf{U}}{\partial x}(x, y)=4 x^{3}-12 x y^{2}, \quad \frac{\partial \mathbf{U}}{\partial y}(x, y)=4 y^{3}-12 x^{2} y, \quad(x, y) \in \Gamma .
$$

The exact solution of this problem is $\mathbf{U}(x, y)=x^{4}-6 x^{2} y^{2}+y^{4}$, so the velocity vector is $(u, v)=\left(4 y^{3}-12 x^{2} y,-4 x^{3}+\right.$ $\left.12 x y^{2}\right)$. Let us compute the approximate solution using the above algorithm. By [5, Lemma 5.1.3] the problem is reduced to (7) with the right-hand side $f(t)=4 x^{3}-12 x y^{2}+i\left(4 y^{3}-12 x^{2} y\right)$. Once an approximate solution $\omega_{n}$ of (2) is obtained from (4), an approximation $\left(\tilde{u}_{i}, \tilde{v}_{i}\right)$ to the velocity field $\left(u_{i}, v_{i}\right)$ is computed via (10) at 90,000 points $\tau_{i}$ on a Cartesian grid on $D$.
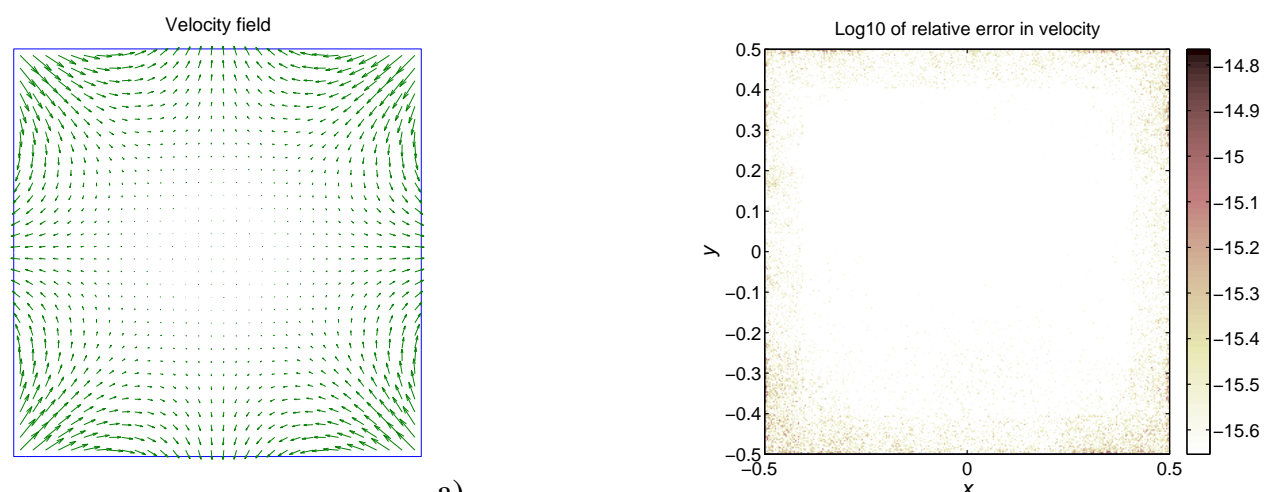

a)

b)

Fig. 2: a presents the actual velocity field $(u, v)$ for the Example 3.1. b shows that the relative pointwise error $\mid\left(u_{i}, v_{i}\right)-$ $\left(\tilde{u}_{i}, \tilde{v}_{i}\right)|/ \max |\left(u_{i}, v_{i}\right) \mid$ is extremely small everywhere in the computational domain. 

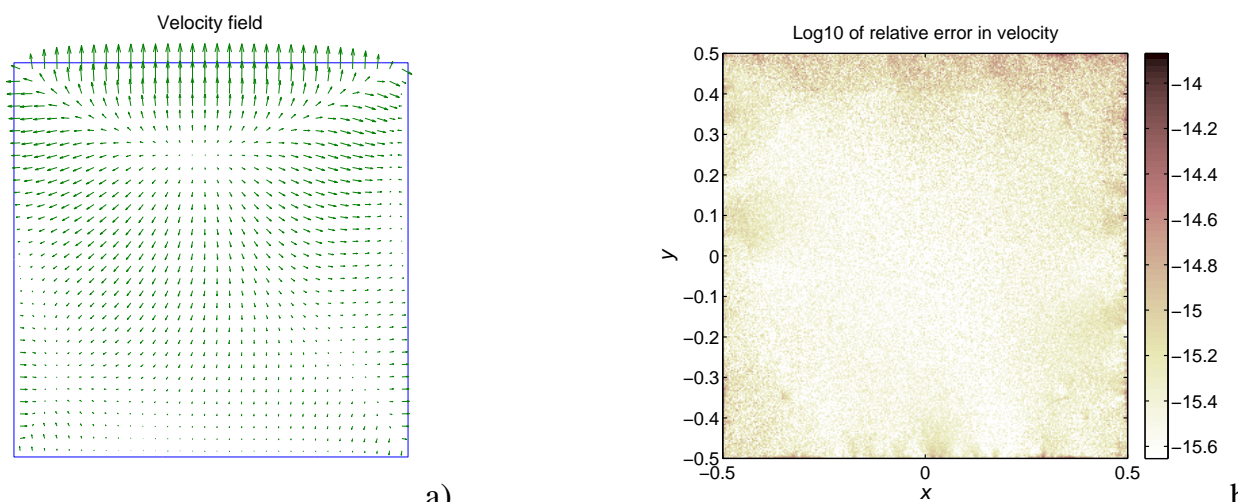

a)

b)

Fig. 3: Same as Example 3.1, but the boundary conditions are discontinuous and given by (11). Only one digit of accuracy is lost compared to the continuous case, cf. Fig. $3 \mathbf{b}$.

Example 3.2 Let $\mathbf{n}$ be the normal to $\Gamma$. Consider the Stokes flow in $D$ described by the following boundary conditions

$$
\mathbf{U}(x, y)=0, \quad \frac{\partial \mathbf{U}}{\partial \mathbf{n}}(x,-0.5)=0, \quad \frac{\partial \mathbf{U}}{\partial \mathbf{n}}(x, 0.5)=1, \quad \frac{\partial \mathbf{U}}{\partial \mathbf{n}}( \pm 0.5, y)=\mp y, \quad(x, y) \in \Gamma .
$$

Following [5, Lemma 5.1.4], one can reduce the problem (11) for (1) to a boundary value problem (7) with the right-hand side (6). Using the previous approach, we obtain approximate values for velocity and vorticity of the corresponding Stokes flow. Results are presented in Fig. 4. The vorticity diverges at the corners and the color bar range is limited to [-15, 15]. The actual values of $\zeta_{i}$ range approximately between -268 and 361 . Fig. $4 \mathbf{b}$ shows estimated absolute errors in the pointwise vorticity $\zeta_{i}$. Taking into account the diverging nature of $\zeta$, this scheme allows us to obtain vorticity almost as accurately as velocity.

Acknowledgements This work started when the first author visited the Research Institute of Mathematical Sciences, Kyoto University, in October, 2013. He would like to thank Professor Hisashi Okamoto for his hospitality and helpful advises.

The authors are also gratefully acknowledge the support from Universiti Brunei Darussalam Grant UBD/PNC2/2/RG/1(159) and from Swedish Research Council Grant 621-2011-5516.

\section{References}

[1] S. G. Mikhlin, Integral equations and their applications to certain problems in mechanics, mathematical physics and technology, Second revised edition. A Pergamon Press Book (The Macmillan Co., New York, 1964).

[2] N. I. Muskhelishvili, Dokl. Akad. Nauk SSSR 3(100), 7-11 (1934).

[3] V.Z. Parton and P. I. Perlin, Integral equations in elasticity ("Mir", Moscow, 1982).

[4] V.D. Didenko and B. Silbermann, J. Comput. Appl. Math. 146(2), 419-441 (2002).

[5] V.D. Didenko and B. Silbermann, Approximation of additive convolution-like operators. Real $C^{*}$-algebra approach, Frontiers in Mathematics (Birkhäuser Verlag, Basel, 2008),

[6] V. D. Didenko and J. Helsing, SIAM J. Numer. Anal 49, 1127-1148 (2011).

[7] V. D. Didenko and J. Helsing, East Asian J. Appl. Math. 1(4), 403-414 (2011).

[8] J. Helsing and R. Ojala, J. Comput. Phys. 227(5), 2899-2921 (2008).

[9] J. Helsing, J. Comput. Phys. 228(23), 8892-8907 (2009).

[10] J. Helsing, Abstr. Appl. Anal. 2013 Article ID 938167 (2013).
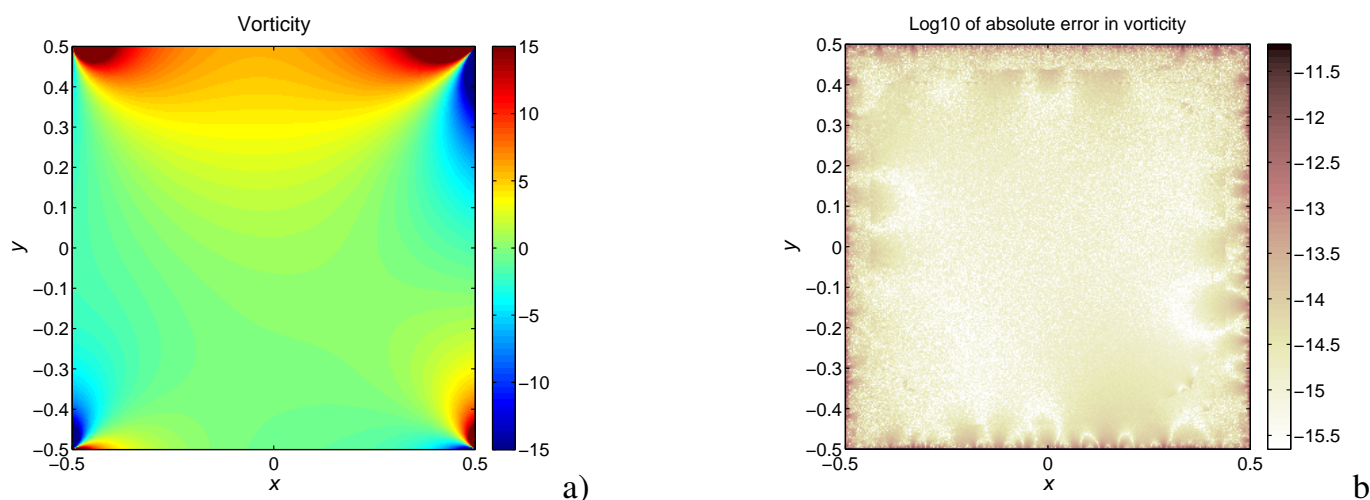

a)

b)

Fig. 4: The vorticity $\zeta=u_{y}-v_{x}$ for the Example 3.2. a illustrates the vorticity $\zeta_{i}$ at 90,000 evaluation points on a Cartesian grid in $D$. 